\title{
Efeito da prática conjunta da caminhada e yoga no sono, depressão e qualidade de vida em pacientes com síndrome da fibromialgia
}

\author{
Effect of joint practice of walking and yoga on sleep, depression, and \\ quality of life in patients with fibromyalgia syndrome
}

\author{
Ricardo de Azevedo Klumb Steffens ${ }^{1}$; Maick da Silveira Viana ${ }^{1}$; Carla Maria de Liz ; \\ Ricardo Brandt ${ }^{2}$; Guilherme Bevilacqua ${ }^{3}$; Alexandro Andrade ${ }^{4}$ \\ ${ }^{1}$ Doutorandos em Ciências do Movimento Humano - Centro de Ciências da Saúde e do Esporte/Universidade do Estado de Santa \\ Catarina - Cefid/Udesc. Florianópolis, SC - Brasil. \\ ${ }^{2}$ Doutor em Ciências do Movimento Humano - Centro de Ciências da Saúde e do Esporte/ Universidade do Estado de Santa \\ Catarina - Cefid/Udesc, Florianópolis, SC. Professor do Colégio de Aplicação - Universidade Federal de Santa Catarina - UFSC. \\ Florianópolis, SC - Brasil. \\ ${ }^{3}$ Graduando em Educação Física - Centro de Ciências da Saúde e do Esporte/Universidade do Estado de Santa Catarina - Cefid/ \\ Udesc. Florianópolis, SC - Brasil. \\ ${ }^{4}$ Professor Doutor do Programa de Pós-Graduação em Ciências do Movimento Humano - Centro de Ciências da Saúde e do Esporte/ \\ Universidade do Estado de Santa Catarina - Cefid/Udesc. Coordenador do LAPE - Laboratório de Psicologia do Esporte e do \\ Exercício. Florianópolis, SC - Brasil.
}

Endereço para correspondência

Ricardo de Azevedo Klumb Steffens

R. Pascoal Simone, 358, Coqueiros

88080-350 - Florianópolis - SC [Brasil]

ricardoaksteffens@gmail.com

\begin{abstract}
Resumo
Introdução: A síndrome da fibromialgia é caracterizada por dores no corpo, e exercícios físicos, como caminhada e yoga, podem ajudar no tratamento. Objetivos: Investigar o efeito da prática conjunta da caminhada e yoga sobre o sono, depressão, impacto da fibromialgia na qualidade de vida, estresse e dor em indivíduos com a síndrome. Métodos: Nove pacientes com média de idade de 53,78 $\pm 5,40$ anos realizaram 32 sessões destes exercícios. Utilizou-se o Questionário Sociodemográfico e Clínico, o Índice de Qualidade do Sono de Pittsburgh, o Questionário de Impacto da Fibromialgia, o Inventário de Depressão de Beck e a Escala de Estresse Percebido. Com o " $\mathrm{t}$ " de Student pareado, verificaram-se as diferenças das variáveis $(p<0,05)$. Resultados: Ocorreram melhoras significativas do sono $(p=0,01)$, diminuição da depressão $(p=0,03)$ e do impacto da fibromialgia na qualidade de vida $(\mathrm{p}=0,04)$. Conclusões: Trinta e duas sessões de prática conjunta de caminhada e yoga produziram melhoras nos sintomas da síndrome.

Descritores: Exercício; Fibromialgia; Ioga; Qualidade de vida; Sono.
\end{abstract}

\begin{abstract}
Introduction: Fibromyalgia syndrome is characterized by body pains and exercise such as walking and yoga could help in the treatment. Objectives: To investigate the effect of joint practice of walking and yoga on sleep, depression, impact of fibromyalgia in quality of life, stress and pain in patients with fibromyalgia syndrome. Methods: Nine patients with mean age of $53.78 \pm 5.40$ years were subjected to 32 sessions of these exercises. We used the Questionnaire Socio-demographic and Clinical, the Quality Index and the Pittsburgh Sleep, the Questionnaire about Fibromyalgia Impact, the Beck Depression Inventory and the Perceived Stress Scale. The paired Student's t-test was used to verify the differences of variables ( $p$ $<0.05)$. Results: There were significant improvements of sleep $(p=0.01)$, decreased of depression ( $p=0.03)$ and the impact of fibromyalgia on quality of life $(p=0.04)$. Conclusions: Thirty-two sessions of joint practice of walking and yoga produced improvements on symptoms of the syndrome.
\end{abstract}

Key words: Exercise; Fibromyalgia; Yoga; Quality of life; Sleep. 


\section{Introdução}

A Síndrome da Fibromialgia (SFM) é uma síndrome complexa e heterogênea com opções limitadas de tratamento, caracterizada por dor crônica, hiperalgesia e sintomas, como fadiga, cansaço matinal, distúrbios de sono e problemas cognitivos. Apesar de intensa pesquisa na área, a patogenia e a etiologia da SFM ainda permanecem em estudo 1 . Conforme o Colégio Americano de Reumatologia (ACR), a SFM é caracterizada por dor musculoesquelética generalizada persistente por mais de três meses, além de pontos anatômicos específicos dolorosos à palpação, denominados tender points, os quais devem ser diagnosticados no mínimo 11 dos 18 pontos mapeados pelo $\mathrm{ACR}^{2}$. A prevalência da SFM varia de $0,66 \%$ a $4,4 \%$ da população ${ }^{3}$. Os pacientes sofrem com uma variedade de sintomas, como ansiedade, depressão, cefaleia, síndrome do cólon irritável, disfunção temporomandibular e síndrome da fadiga crônica ${ }^{4}$. Isso demonstra a complexidade da SFM, confirmando ser uma desordem heterogênea ${ }^{1}$. Atualmente, estudos têm demonstrado os benefícios da prática de exercícios físicos no tratamento da SFM, tais como a prática de caminhada ${ }^{5,6,7,8}$ e do yoga $a^{9,10,11}$. Essas formas de tratamento promovem aos praticantes uma abordagem não farmacológica, de fácil aplicação e de baixo custo para o controle da SFM.

Justifica-se este estudo para uma análise mais aprofundada da prática conjunta de caminhada e yoga em pacientes com SFM, pois verifica-se na literatura que exercícios físicos realizados com mais de uma modalidade têm um efeito positivo sobre a síndrome. Com isso, teve como objetivo verificar o efeito da prática conjunta de 32 sessões de caminhada e yoga sobre a qualidade do sono, depressão, impacto da fibromialgia na qualidade de vida, estresse e dor de mulheres com síndrome da fibromialgia.

\section{Material e métodos}

Esta pesquisa caracteriza-se como sendo um estudo clínico com delineamento de me- didas pré e pós-tratamento. O estudo foi conduzido dentro dos padrões éticos exigidos pela Declaração de Helsinque de 1964 e de acordo com a Resolução 196/96 do Conselho Nacional de Saúde. O projeto foi aprovado pelo Comitê de Ética em Pesquisa em Seres Humanos (CEP) da Universidade do Estado de Santa Catarina, sob o número de referência 103/2010.

Os participantes do estudo foram escolhidos de maneira não probabilística intencional, sendo nove mulheres, com média de idade de $53,78 \pm 5,40$ anos, que realizaram 32 sessões conjuntas de caminhada e yoga. O critério de inclusão foi morar na região da grande Florianópolis, ter diagnóstico clínico de síndrome da fibromialgia, estar apto para a prática de exercícios físicos, não possuir doenças que impedissem a participação no programa de tratamento (como doenças cardíacas e diagnóstico de problemas na coluna). Todas as participantes do estudo estavam inscritas no programa de Extensão "Psicologia do Esporte e do Exercício Aplicada à Saúde", do Centro de Ciências da Saúde e do Esporte - Cefid, da Universidade do Estado de Santa Catarina - Udesc. As voluntárias eram sedentárias antes do início da pesquisa e todas as nove participaram do começo ao fim desta.

Os seguintes instrumentos foram utilizados no trabalho:

a) Questionário Sociodemográfico e Clínico, adaptado de Konrad ${ }^{12}$, composto por variáveis sociodemográficas - idade, estado civil, escolaridade, ocupação e renda familiar - e por variáveis clínicas - tempo dos sintomas, tempo do diagnóstico, tratamentos utilizados, uso de medicação, fatores causais, sintomas, fatores moduladores e outras condições clínicas associadas à SFM.

b) Índice da Qualidade do Sono de Pittsburgh (PSQI) desenvolvido por Buysse et al. ${ }^{13}$, validado por Konrad ${ }^{12}$ na forma curta. A partir de nove questões, este questionário avalia a qualidade e padrão do sono de adultos. Os resultados são agrupados em sete componentes, tais como qualidade 
subjetiva, latência, duração, eficiência habitual, distúrbios, uso de medicação e disfunções diurnas.

c) Inventário de Depressão de Beck, traduzido e validado por Gorestein e Andrade ${ }^{14}$. É composto de 21 itens com uma escala de zero a três, que verifica a intensidade do sintoma. O escore máximo é 63 (maior nível de depressão). Somados todos os itens, este inventário fornece uma pontuação final que pode variar de zero a 63 pontos, e quanto maior a pontuação, maior o indicativo de sintomas depressivos.

d) Questionário de Impacto da Fibromialgia (QIF), validado no Brasil por Marques e Santos $^{15}$ avalia o impacto da SFM sobre a qualidade de vida por meio de dez componentes: capacidade funcional, bem-estar, faltas no trabalho, capacidade de trabalhar, dor, fadiga, cansaço matinal, rigidez, ansiedade e depressão.

e) Escala de Estresse Percebido, validada por Luft et al. ${ }^{16}$ é uma escala composta por 14 subescalas do tipo Likert, nas quais o participante assinala uma opção que varia de zero a quatro para cada uma, podendo variar de zero (sem estresse) a 56 (extremo estresse).

f) Escala Visual Analógica - EVA ${ }^{15}$ foi usada na avaliação da intensidade da dor.

Inicialmente, as voluntárias do estudo foram convidadas a participar de uma entrevista, na qual foram relatadas as informações referentes à SFM, à prática de caminhada e yoga, à pesquisa, aos objetivos, à relevância e procedimentos das avaliações. Em seguida, as que aceitaram participar das práticas e avaliações assinaram o Termo de Consentimento Livre e Esclarecido. Posteriormente, responderam ao questionário de caracterização sociodemográfica e clínica e aos questionários do pré-teste, com os quais se avaliaram qualidade do sono, depressão, impacto da fibromialgia na qualidade de vida, estresse e dor de mulheres com SFM. Essas avaliações foram realizadas previamente às práticas propos- tas no estudo e após as 32 sessões de caminhada e yoga previstas.

Realizaram-se 32 sessões de caminhada, com duração de 45 minutos cada, duas vezes por semana, durante o intervalo de quatro meses. Cada sessão foi dividida nas seguintes fases: (a) 10 minutos de aquecimento, com exercícios de alongamento e aquecimento para as principais articulações do corpo; (b) 25 minutos de caminhada; (c) 10 minutos de relaxamento, sendo realizados exercícios respiratórios e alongamentos com o objetivo de diminuir a frequência cardíaca e possibilitar o retorno aos níveis de repouso do organismo.

A prescrição da intensidade do treinamento usada durante a prática da caminhada foi estabelecida após a realização de testes ergoespirométricos, sendo utilizada a frequência cardíaca do limiar anaeróbio (LA), ou logo abaixo dela, pois indica uma intensidade adequada para ganho de aptidão física, com maior segurança e mais adesão dos pacientes às práticas de exercícios físicos. Os testes ergoespirométricos foram realizados pelo seguinte protocolo: (a) repouso: em posição ortostática, sendo efetuado um eletrocardiograma com aparelho da marca TEB, com o objetivo de avaliação cardiovascular em repouso; (b) esforço físico: realizado teste em esteira rolante, por intermédio de um analisador de gases, em que foi verificado o consumo de oxigênio (valor mais elevado do consumo máximo de oxigênio alcançado durante o teste). Usou-se uma esteira da marca Imbramed ATL 15000, Porto Alegre, Brasil; e para verificação do $\mathrm{VO}_{2}$ máximo, um equipamento Córtex Biophisik, Leipzig, Alemanha. A velocidade inicial do teste começou com 1,7 mph e terminou com $5 \mathrm{mph}$, e a inclinação iniciou com zero e finalizou com 15\%. O objetivo desse teste foi determinar a avaliação cardiovascular durante o exercício e também do limiar anaeróbio para a prescrição durante a prática de exercícios físicos. No máximo dois dias após a realização do teste, o paciente começou a prática de exercícios físicos.

A intervenção da prática de yoga durou 45 minutos, seguindo o protocolo de intervenção: 
filosofia do yoga, execução de exercícios respiratórios, execução de posturas psicofísicas e prática de meditação/relaxamento, conforme detalhado na Figura 1.

\begin{tabular}{|c|c|}
\hline $\begin{array}{c}\text { Filosofia } \\
\text { do yoga } \\
\text { (5 minutos) }\end{array}$ & $\begin{array}{c}\text { Realizou-se leitura e discussão sobre a } \\
\text { filosofia do yoga. }\end{array}$ \\
\hline $\begin{array}{c}\text { Exercícios } \\
\text { respiratórios } \\
\text { (10 minutos) }\end{array}$ & $\begin{array}{c}\text { Momento para concentração } \\
\text { (prathyahara) e execução de exercícios } \\
\text { respiratórios (pranayamas). }\end{array}$ \\
\hline $\begin{array}{c}\text { Posturas } \\
\text { psicofísicas } \\
\text { (25 minutos) }\end{array}$ & $\begin{array}{c}\text { Realizadas dez posturas psicofísicas } \\
\text { (ásas), de acordo com a individualidade } \\
\text { dois minutos. Foi realizada respiração } \\
\text { profunda e consciente durante a } \\
\text { permanência na postura. }\end{array}$ \\
\hline $\begin{array}{c}\text { Prática de } \\
\text { meditação/ } \\
\text { relaxamento } \\
\text { (5 minutos) }\end{array}$ & $\begin{array}{c}\text { Realizada na postura shavásana (posição } \\
\text { deitada em decúbito dorsal) a técnica de } \\
\text { relaxamento consciente do corpo físico } \\
\text { e da mente (yoga nidra), objetivando } \\
\text { maior integração e consciência corporal, } \\
\text { proporcionando a recuperação do esforço. }\end{array}$ \\
\hline
\end{tabular}

Figura 1: Protocolo de intervenção da prática de yoga no estudo realizado

Os dados foram analisados estatisticamente por meio de análise descritiva e inferencial. Realizou-se análise descritiva exploratória dos dados por meio da verificação de médias, frequências, percentagens e desvios-padrão.

Foi realizado o teste de Shapiro-Wilk para verificar se as variáveis apresentavam distribuição normal, e estas se caracterizaram como sendo paramétricas e não paramétricas. Para verificar as diferenças das médias das variáveis dependentes (pré- e pós-teste), foi utilizado o teste de Wilcoxon para os dados de distribuição não normal e foi utilizado o teste " $\mathrm{t}$ " de Student pareado para os dados de distribuição normal. Estabeleceu-se como nível de significância para os testes estatísticos $(p<0,05)$.

\section{Resultados}

Os resultados dos dados sociodemográficos e clínicos aparecem na Tabela 1 .
Tabela l: Dados sociodemográficos e clínicos dos participantes do estudo

\begin{tabular}{|c|c|}
\hline Variável & $\begin{array}{c}\text { Grupo }(\mathrm{n}=9) / \\
\text { Média/DP }\end{array}$ \\
\hline Idade (anos) & $53,78 \pm 5,40$ \\
\hline \multicolumn{2}{|l|}{ Gênero } \\
\hline Feminino & $9(100 \%)$ \\
\hline Masculino & 0 \\
\hline \multicolumn{2}{|l|}{ Estado civil } \\
\hline Casada & $3(33,3 \%)$ \\
\hline Não casada (solteira, viúva, separada) & $6(66,7 \%)$ \\
\hline \multicolumn{2}{|l|}{ Nível educacional } \\
\hline Fundamental completo & $1(11,1 \%)$ \\
\hline Médio completo & $5(55,6 \%)$ \\
\hline Médio incompleto & $1(11,1 \%)$ \\
\hline Superior incompleto & $2(22,2 \%)$ \\
\hline \multicolumn{2}{|l|}{ Ocupação atual } \\
\hline Trabalha formalmente & $4(44,4 \%)$ \\
\hline Não trabalha atualmente & $5(55,6 \%)$ \\
\hline \multicolumn{2}{|l|}{ Tempo do início dos sintomas (meses) } \\
\hline 1 a 24 meses & $2(22,2 \%)$ \\
\hline 25 a 60 meses & $1(11,1 \%)$ \\
\hline Mais de 61 meses & $6(66,7 \%)$ \\
\hline \multicolumn{2}{|c|}{ Causas associadas ao aparecimento da SFM } \\
\hline Episódio emocional & $5(55,6 \%)$ \\
\hline Depressão & $4(44,4 \%)$ \\
\hline Esforço físico exagerado & $4(44,4 \%)$ \\
\hline \multicolumn{2}{|l|}{ Sintomas mais presentes } \\
\hline Dor generalizada & $7(77,8 \%)$ \\
\hline Rigidez articular & $6(66,7 \%)$ \\
\hline Sono não restaurador & $6(66,7 \%)$ \\
\hline Cansaço & $6(66,7 \%)$ \\
\hline Fadiga & $5(55,6 \%)$ \\
\hline Dor de cabeça frequente & $5(55,6 \%)$ \\
\hline Mãos e pernas inquietas & $5(55,6 \%)$ \\
\hline Falha de memória & $4(44,4 \%)$ \\
\hline Ansiedade excessiva & $4(44,4 \%)$ \\
\hline Dificuldade de concentração & $4(44,4 \%)$ \\
\hline Tontura & $3(33,3 \%)$ \\
\hline Formigamento & $2(22,2 \%)$ \\
\hline Mau humor & $2(22,2 \%)$ \\
\hline
\end{tabular}

Após as 32 sessões da prática conjunta de caminhada e yoga (Tabela 2), as participantes da pesquisa apresentaram uma melhora significativa na qualidade do sono ( $\mathrm{p}=0,01)$, na depressão $(p=0,03)$ e no impacto da fibromialgia na qualidade de vida $(p=0,04)$. Não ocorreram reduções significativas do estresse $(p=0,32)$ e da intensidade da dor $(p=0,06)$. 
Tabela 2: Efeito da caminhada e yoga na qualidade do sono, depressão, impacto da fibromialgia na qualidade de vida, estresse e dor de mulheres com SFM, após 32 sessões $(n=9)$

\begin{tabular}{lccc}
\hline \multicolumn{1}{c}{ Variável } & $\begin{array}{c}\text { Média } \\
\text { pré-teste }( \pm)\end{array}$ & $\begin{array}{c}\text { Média } \\
\text { pós-teste }( \pm)\end{array}$ & p \\
\hline Qualidade do sono & $12,56(4,9)$ & $8,22(4,2)^{*}$ & 0,01 \\
Depressão & $13,33(6,3)$ & $7,44(7,0)^{*}$ & 0,03 \\
$\begin{array}{l}\text { Impacto da fibromialgia } \\
\text { na qualidade de vida }\end{array}$ & $57,58(25,1)$ & $34,82(19,0)^{*}$ & 0,04 \\
$\quad \begin{array}{llll}\text { Capacidade } \\
\text { funcional }\end{array}$ & $4,26(2,94)$ & $3,01(1,99)$ & 0,30 \\
Bem estar & $6,03(2,84)$ & $4,60(2,34)$ & 0,32 \\
Faltas no trabalho & $2,54(3,25)$ & $2,70(3,23)$ & 0,91 \\
$\quad$ Capacidade de & $5,33(2,52)$ & $3,12(2,81)$ & 0,94 \\
trabalhar & $6,50(3,22)$ & $3,80(2,30)$ & 0,06 \\
Dor & $7,50(3,31)$ & $4,58(2,76)$ & 0,58 \\
Fadiga & $7,12(3,66)$ & $4,92(2,95)$ & 0,28 \\
Cansaço matinal & $6,00(3,07)$ & $5,15(2,39)$ & 0,57 \\
Rigidez & $6,10(3,56)$ & $3,78(3,20)$ & 0,06 \\
Ansiedade & $6,23(3,40)$ & $2,74(2,23)$ & $0,01^{*}$ \\
Depressão & $24,77(9,0)$ & $21,77(7,4)$ & 0,32 \\
\hline Estresse & & & \\
\hline
\end{tabular}

*Diferença significativa ao nível de $p \leq 0,05$.

\section{Discussão}

As participantes deste estudo apresentaram média de idade de 53,78 $\pm 5,40$ anos, similar ao estudo de Berber et al. ${ }^{17}$, no qual predominou a faixa etária de 40 a 55 anos. Em três trabalhos, em que foi realizada a prática de caminhada em pacientes com fibromialgia, predominaram as médias de idade de 48 anos $^{6}, 49 \operatorname{anos}^{7}$ e 54 anos $^{8}$. Cavalcante et al. ${ }^{3}$ identificaram que os sintomas da SFM costumam surgir especialmente a partir dos 35 anos de idade. Parece claro, pela literatura, que a faixa etária predominante da SFM é entre 40 e 55 anos, idade em que começam também a aparecer outras doenças. Um fator também relevante é o diagnóstico tardio, que somente pode ser comprovado nesta faixa etária, mas os sintomas podem ter surgido há mais tempo.
As informações referentes ao nível educacional foram similares aos resultados encontrados em estudos desenvolvidos por Berber ${ }^{17} \mathrm{e}$ Konrad $^{12}$, em que a maioria dos indivíduos com SFM possuíam ensino médio completo e superior incompleto. Percebe-se na literatura uma variação em relação ao nível educacional, e mais estudos são necessários para obter-se uma afirmativa mais concreta sobre o tema.

Em relação à ocupação, em pesquisas anteriores ${ }^{12,17}$ demonstraram que aproximadamente $40 \%$ da amostra eram donas de casa, precisamente igual à porcentagem encontrada neste estudo. Em outro trabalho realizado ${ }^{8}$, verificou-se que $18,2 \%$ exerciam a profissão no momento da pesquisa, $18,2 \%$ estavam afastados do trabalho, $18,2 \%$ não estavam exercendo a profissão atualmente e $45,4 \%$ estavam aposentados. A SFM causa frequentes afastamentos do trabalho principalmente por causa das dores, trazendo um enorme prejuízo tanto econômico quanto social.

Quanto ao início dos sintomas, o tempo médio observado em um estudo ${ }^{8}$ foi quatro anos e seis meses, similar ao estudo aqui apresentado, no qual ocorreu um predomínio de mais de cinco anos, enfatizando que o diagnóstico é realizado numa fase posterior ao início dos sintomas, como já citado anteriormente.

O início da SFM frequentemente parece ser seguido de um estresse físico ou psicológico. Anderberg et al. ${ }^{18}$ afirmam que, comparados com indivíduos saudáveis, há evidência de que aqueles com SFM sofrem eventos estressantes na vida precocemente. Neste estudo, em relação às causas associadas ao aparecimento da SFM, constataram-se episódios emocionais, depressão e esforço físico exagerado como causadores da SFM, confirmando, dessa forma, o trabalho acima citado.

Os sintomas mais frequentes relatados pelas participantes deste estudo obtiveram concordância com outras pesquisas, tais como a de Helfenstein e Feldman ${ }^{19}$, em que a dor generalizada é apontada como principal característica da síndrome e como pré-requisito para seu diagnóstico, conforme os critérios propostos pelo 
$\mathrm{ACR}^{2}$. A ocorrência destes principais sintomas aparece na maioria dos pacientes com SFM.

No estudo aqui mostrado, após 32 sessões de caminhada e yoga, ocorreu uma melhora significativa na qualidade do sono. Gui et al. ${ }^{20}$ afirmam que intervenções destinadas a melhorar a qualidade do sono podem trazer benefícios à saúde e qualidade de vida para pacientes com SFM, sendo relevante a avaliação do sono e pesquisas na busca por tratamento adequado aos distúrbios do sono nesses pacientes. Em estudo anterior de Steffens et al. ${ }^{6}$ em que foram realizadas também 32 sessões de caminhada orientada, durante quatro semanas, similar a este trabalho, verificou-se uma melhora significativa na qualidade do sono do grupo analisado. Resultado positivo no sono ocorreu também no estudo de Pasqua et al. ${ }^{8}$, em que, após oito sessões de caminhada realizadas durante um mês, observouse alteração positiva, de forma significante, na qualidade subjetiva e na percepção dos distúrbios do sono nos participantes com síndrome da fibromialgia. Quanto aos outros componentes estudados, verificou-se que melhorou a latência, a duração, a eficiência habitual e as disfunções diurnas, porém de forma não significante. Neste último estudo, apesar de ter ocorrido apenas oito sessões, o que é considerado pela literatura um número pequeno, identificou-se um efeito positivo no sono das pacientes. Por estes estudos, verifica-se que a variável sono parecer reagir positivamente a um tratamento com exercícios físicos em pacientes com SFM, pois mesmo com pouco tempo de prática já parece ocorrer um efeito positivo.

Referente à depressão, observou-se uma redução, neste estudo, diferente de um anterior ${ }^{6}$, em que a prática de 32 sessões de caminhada orientada, realizada duas vezes por semana, não modificou significativamente essa doença. Já Woolery et al. ${ }^{21}$ realizaram um estudo com 28 sujeitos saudáveis, com idade entre 18 e 29 anos e níveis moderados de depressão, os quais participaram de duas aulas semanais de yoga, com duração de 60 minutos, durante cinco semanas e ocorreu a diminuição da depressão dos parti- cipantes. Parece que a variável depressão ainda apresenta discordâncias entre os estudos, e sugerem-se mais pesquisas sobre o tema para que se possam ter resultados mais conclusivos.

Em relação ao impacto da SFM na qualidade de vida, verificou-se uma diminuição deste, melhorando a qualidade de vida dos pacientes estudados. No estudo de Santos e Santos ${ }^{10}$, ocorreu também uma diminuição do impacto da SFM na qualidade de vida de sete mulheres com SFM que realizaram um programa de Kundalini Yoga, durante oito semanas, com duas aulas semanais e duração de uma hora e dez minutos. $\mathrm{Na}$ pesquisa de Silva et al. ${ }^{22}, 17$ mulheres com SFM realizaram oito sessões de yoga, com duração de 50 minutos cada sessão, durante quatro semanas e, como resultado, ocorreu a diminuição do impacto da SFM na qualidade de vida. Em trabalho realizado ${ }^{7}$ com oito mulheres com SFM que realizaram dez sessões de prática de caminhada, foi verificado que o impacto da SFM na qualidade de vida destas pacientes não sofreu alteração significativamente estatística. O fato de haver ocorrido um pequeno número de sessões (10) e somente cinco semanas de prática pode ter prejudicado os resultados desse estudo citado anteriormente, ao contrário deste atual trabalho, em que com 32 sessões de prática conjunta de caminhada e yoga, realizadas em 16 semanas proporcionaram resultados significativos. Percebe-se que o tempo de prática mais longo pode gerar um efeito mais positivo sobre a qualidade de vida em pacientes com SFM.

Na relação do estresse e SFM, Becker et al. ${ }^{23}$ verificaram que $95 \%$ das mulheres com SFM se enquadraram em algum nível de estresse, comparadas com $51,2 \%$ da amostra controle com algum nível de estresse. Smith et al. ${ }^{24}$ realizaram um estudo randomizado, com 66 pessoas, que realizaram a prática de yoga, por dez semanas, com uma sessão semanal de uma hora de prática em cada. Após a décima semana, houve diminuição do estresse, o que foi um fato importante pelo reduzido número de sessões (10) efetuadas, situação contrária ao atual estudo, em que apesar de uma quantidade maior de sessões (32) não 
ocorreu uma melhora significativa dessa variável, possivelmente em razão do nível mais baixo de estresse inicial das participantes do estudo.

Em relação ao efeito sobre a dor, Carson et al. ${ }^{11}$ analisaram o efeito da prática de yoga em 22 mulheres com SFM, durante dois meses, com uma aula realizada por semana com duração de 120 minutos. Os resultados desse estudo mostraram melhoras significativas na funcionalidade e diminuição dos sintomas, como dor e fadiga. No estudo de Matsutani et al. ${ }^{25}$ foram comparados 12 pacientes com SFM, que realizaram a prática de 45 minutos alongamentos, e sete pacientes, que realizaram 30 minutos de prática de caminhada em esteira ergométrica, ambos efetuados em oito sessões e com frequência semanal de uma vez por semana. Os resultados mostram que o grupo que realizou alongamento apresentou redução na intensidade da dor, número de tender points, limiar de dor em quatro tender points $(\mathrm{p}<0,05)$. O grupo que fez caminhada na esteira mostrou piora no limiar de dor de seis tender points. Na pesquisa atual, apesar de ter ocorrido uma diminuição da dor esta não se mostrou significativa, demonstrando que essa variável ainda carece de estudos mais aprofundados, pois é o principal sintoma da fibromialgia e, provavelmente, o que pode demorar mais a sofrer alterações.

\section{Conclusões}

Verificou-se que 32 sessões de prática conjunta de caminhada e yoga produziram efeitos positivos na melhora da qualidade do sono, diminuições na depressão e no impacto da fibromialgia na qualidade de vida de mulheres com a síndrome. Não ocorreram alterações significativas do estresse e da intensidade da dor.

\section{Agradecimentos}

Os autores agradecem à Universidade do Estado de Santa Catarina - Udesc, pela opor- tunidade do estudo, à Fundação de Amparo à Pesquisa e Inovação do Estado de Santa Catarina - Fapesc, por apoio financeiro por meio de edital de pesquisa (Projeto $n^{\circ}$ 2442-2011/12), à Coordenação de Aperfeiçoamento de Pessoal de Nível Superior - Capes, pela bolsa de pesquisa concedida a pesquisador participante do estudo e ao Conselho Nacional de Desenvolvimento Científico e Tecnológico - CNPq, editais $\mathrm{n}^{\circ}$ 10/2010 e 14/2012, pela bolsa de apoio técnico a pesquisador participante do artigo.

\section{Referências}

1. Rehm SE, Koroschetz J, Gockel U, Brosz M, Freynhagen R, Tölle TR, et al. A cross-sectional survey of 3035 patients with fibromyalgia: subgroups of patients with typic comorbidities and sensory symptom profiles. Rheumatology. 2010;49(6):1146-52.

2. Wolfe F, Smythe HA, Yunus MB, Bennett AM, Bombardier CE, Goldenberg DL, et al. The American College of Rheumatology 1990. Criteria for the classification of Fibromyalgia: Report of the multicenter criteria committee. Arthritis Rheum. 1990;33(2):160-72.

3. Cavalcante AB, Sauer JF, Charlot SD, Assumpção A, Lage LV, Matsutani LA, et al. A prevalência de fibromialgia: uma revisão de literatura. Rev Bras Reumatol. 2006;46(1):40-8.

4. Calandre EP, Garcia CJ, Garcia LJM, Rico VF, Molina BR, Rodriguez LCM, et al. Subgrouping patients with fibromyalgia according to the results of the fibromyalgia impact questionnaire: a replication study. J Rheumatol. 2011;31(12):1555-9.

5. Meyer BB, Lemley KJ. Utilizing exercise to affect the symptomology of fibromyalgia: a pilot study. Med Sci Sports Exerc. 2000;32(10):1691-7.

6. Steffens RAK, Liz CM, Viana MS, Brandt R, Oliveira LGA, Andrade A. Praticar caminhada melhora a qualidade do sono e os estados de humor em mulheres com síndrome da fibromialgia. Rev Dor. 2011;12(4):327-31.

7. Steffens RAK, Vale BR, Fonseca ABP, Viana MS, Brandt R, Andrade A. Efeito da caminhada sobre a qualidade de vida e auto-eficácia de mulheres com síndrome da fibromialgia. Rev Bras Ciênc Mov. 2012;20(1):41-6. 
8. Pasqua LD, Steffens RAK, Viana MS, Brandt R, Vilarino GT, Andrade A. Efeito da caminhada no sono de pacientes portadores de síndrome da fibromialgia. ConScientiae Saúde. 2012;11(4):580-6.

9. Silva GD, Lage LV. Ioga e fibromialgia. Rev Bras Reumatol. 2006;46(1):37-9.

10. Santos LAL, Santos DL. A influência da prática regular de kundalini yoga sobre variáveis funcionais em indivíduos portadores da síndrome da fibromialgia. Rev Bras Ciênc Mov. 2008;16(2):7-15.

11. Carson JW, Carson KM, Jones KD, Bennett RM, Wright CL, Mist SD. A pilot randomized controlled trial of the yoga of awareness program in the management of fibromyalgia. Pain. 2010;151(2):530-9.

12. Konrad LM. Efeito agudo do exercício físico sobre a qualidade de vida de mulheres com síndrome da fibromialgia [dissertação de mestrado em Educação Física]. Florianópolis: Centro de Desportos, Universidade Federal de Santa Catarina; 2005.

13. Buysse DJ, Reynolds CF, Monk TH, Berman SR, Kupfer DJ. The Pittsburgh Sleep Quality Index: a new instrument for psychiatric practice and research. Psychiatry Res.1989;28(2):193-213.

14. Gorenstein C, Andrade L. Validation of a Portuguese version of the Beck Depression Inventory and the State-Trait Anxiety Inventory in Brazilian subjects. Braz J Med Biol Res. 1996;29(4):453-7.

15. Marques AP, Santos AMB, Assumpção A, Matsuttani LA, Lage LG, Pereira CAB. Validação da versão brasileira do Fibromyalgia Impact Questionnaire (FIQ). Rev Bras Reumatol. 2006;46(1):24-31.

16. Luft CD, Sanches SO, Mazo GZ, Andrade A. Versão brasileira da Escala de Estresse Percebido: tradução e validação para idosos. Rev Saúde Pública. 2007;41(4):606-15.
17. Berber JSS, Kupek E, Berber SC. Prevalência de depressão e sua relação com a qualidade de vida em pacientes com síndrome da fibromialgia. Rev Bras Reumatol. 2005;45(2):47-54.

18. Anderberg UM, Mareinsdottir I, Theorell T, von Knorring L. The Impact of life events in female patients with fibromyalgia and in female healthy controls. Eur Psychiatry. 2000;(15):295-301.

19. Helfenstein M, Feldman, D. Síndrome da fibromialgia: características clínicas e associações com outras síndromes disfuncionais. Rev Bras Reumatol. 2002;42(1):8-14.

20. Gui M, Pedroni CR, Rossini S, Reimão R, Barbosa CMR. Distúrbios do sono em pacientes com fibromialgia. Neurobiologia. 2010;73(1):175-82.

21. Woolery A, Myers H, Sternlieb B, Zeltzer L. A yoga intervention for young adults with elevated symptoms of depression. Altern Ther Health Med. 2004;10(2):60-3.

22. Silva G, Lorenzi-Filho G, Lage LV. Effects of Yoga and the addition of Tui Na in patients with fibromyalgia. J Altern Complement Med. 2007;13(10):1107-13.

23. Becker RMR, da Silva VK, Machado FS, dos Santos AF, Meireles DC, Mergener M, et al. Interação entre qualidade do meio ambiente, estresse e a variação do gene APOE na determinação da suscetibilidade à fibromialgia. Rev Bras Reumatol. 2010;50(6):617-24.

24. Smith C, Hancock H, Blake-Mortimer J, Eckert K. A randomised comparative trial of yoga and relaxation to reduce stress and anxiety. Complement Ther Med. 2007;15(2):77-83

25. Matsutani LA, Assumpção A, Marques AP. Exercícios de alongamento muscular e aeróbico no tratamento da fibromialgia: estudo piloto. Fisioter Mov. 2012;25(2):411-8. 